\title{
A Quantification Method in Quadrupole Mass Spectrometer Measurement
}

\author{
Received 15 February, 2021; accepted 3 March, 2021
}

\author{
Young Seok Lee ${ }^{a}$, Sang Hyeop Oh ${ }^{c}$, Jang Jae Lee ${ }^{a}$, Chul Hee Cho ${ }^{a}$, Si Jun Kim ${ }^{d}$, and Shin Jae You ${ }^{a, b, *}$ \\ a Department of Physics, Chungnam National University, Daejeon 34134, Republic of Korea \\ bInstitute of Quantum Systems (IQS), Chungnam National University, Daejeon 34134, Republic of Korea \\ cKorea Research Institute of Standards and Science, Daejeon 34113, Republic of Korea \\ dNanotech Optoelectronics Research Center, Yongin 16882, Republic of Korea
}

*Corresponding author E-mail: sjyou@cnu.ac.kr

\begin{abstract}
Quadrupole mass spectrometry (QMS) has been widely used in the semiconductor industry to monitor plasma radical or ion species, but the signal used in QMS is mostly not quantified. To quantify QMS signals to radical density measurements, the signals should be calibrated by a reference. During this calibrating process, it is important to apply a proper signal proportional coefficient between the signal of QMS and the particle density of the plasma, which is known to be strongly related to the mass of the particle, but is not precisely well known one until now. In this work, we propose a simple approach to obtain reliable signal proportional coefficients of different masses by using with standard gases, which are precisely specified components through gas manufacturing. This approach is expected to raise the reliability of quantifying QMS data, contributing to an advance in plasma database development.
\end{abstract}

Keywords: Quadrupole mass spectrometry, Plasma, Plasma diagnostics, Standard gas

\section{Introduction}

Plasma processing in manufacturing semiconductor devices has attracted enormous interest due to its various advantages $[1,2]$. Especially, in the etching process, plasma has played an essential role in fabricating fine structures on wafers for decades [3-6]. The manufacturing high-aspect-ratio features of NAND flash memory devices, for example, has a challenge to etch the substrate through narrow and deep holes patterned on the mask, for which plasma provides radical neutrals and bombarding ions to the substrate, enabling highly anisotropic etching to be feasible $[7,8]$. In these processes, it has been shown that plasma radicals and ions have a synergetic effect on etching, namely that chemical reactions between radicals and the substrate are enhanced by energetic ion bombardment [9]. Therefore, knowing radical and ion information such as their density, distribution, etc. is highly important to control and optimize the process [10].

There are various methods (and equipment) to measure radical and ion densities. Optical emission spectrometry provides radical species information by analyzing the light emitted from de-excited radicals that has different wavelengths according to the electron energy levels of each species $[11,12]$. A retarding field energy analyzer is widely used for measuring ion energy distribution as well as ion density, and it employs multiple electrodes where different bias voltages are properly applied so that electrons are repelled and ions are filtered according to their energy [13]. One of the most powerful equipment is a quadrupole mass spectrometer (QMS) that, as its name implies, measures radical densities according to their mass using a quadrupole electrode [14]. These devices are widely used in semiconductor industry because they are compact, easy to use, and cost-effective.

However, there are some challenges to obtain precisely quantified radical densities because QMS signals represent only the partial pres- sures of each species having different masses. Thus, there has been many studies reporting methods to convert QMS signals to absolute radical density values in plasma [15-17]. low:

Singh et al. suggested that a QMS signal can be represented as be-

$$
S=\alpha I_{e} \sigma_{i} n
$$

where $S$ is the measured QMS signal intensity and $\alpha$ represents the signal measurement efficiency (ME) that is a function of the mass-tocharge ratio of each species generated in the QMS ionizer. $I_{e}$ is the emission current flowing through the filament in the ionizer, $\sigma_{i}$ is the ionization cross section of the target species, and $n$ is the number density of the target particle inside the ionizer. The ME $\alpha$ is the product of the extraction efficiencies of the ions from the ionizer, the transmission efficiencies of the quadrupole mass filter that depends on the species mass-to-charge ratio, the detection efficiencies of the channeltron detector that also depends on the species mass-to-charge ratio, and lastly the length of the ionizer cage [17].

A measured QMS signal represented as above is calibrated with a reference signal to eliminate the unknown parameters such as the filament emission current, and then quantified to absolute particle number densities [18]. In this process, it is important that the ME is properly determined for each QMS since the difference between equipment geometries as well as ionizer structures causes various ion extraction efficiencies from the ionizer, transmission efficiencies in the quadrupole mass filter, or detection efficiencies of the Faraday cup detector. Kessels et al. suggested that the transmittance of the mass filter in their device was best estimated as a decrease with ion mass inversely proportional to the square root of the mass, and also that the detection efficiency of the channeltron decreases similarly, leading to an overall mass discrimination inversely proportional to the ion mass [15]. However, this mass dependence of the ME proposed by Kessels et al. 
was not derived with rigorous theoretical analysis so that employing it directly to one's QMS data quantification can cause inevitable errors. Singh et al. concluded that the optimum mass dependence of the measurement efficiency of their instrument was represented as the ion mass to the power of -0.81 , which was obtained by fitting their experimental data [17].

In this paper, we propose a method to obtain a proper ME for high-reliability quantification of QMS data using a standard gas, which refers to a mixed gas in which each proportion of every component is exactly specified. Standard gases can be useful to determine an ME in QMS measurements since it has various gas species with different masses, allowing the ion mass dependence of an ME to be easily estimated in a wide range of ion masses. Moreover, the specific component information of a standard gas can provide useful reference data for calibrating QMS signals since one can calculate the absolute density of each component by the ideal gas law, all of which can be used as a reference signal for the calibration. Our approach is expected to make QMS data processing easier as well as improve the reliability of plasma QMS diagnostics. Our experimental setup and details are described in the next section, followed by a description of the new approach, discussion, and conclusions.

\section{Experimental details}

As shown in Fig. 1, the vacuum chamber used for the experiments has a cylindrical structure; the diameter is $340 \mathrm{~mm}$ and the height is $145 \mathrm{~mm}$. This geometrical information is used when we calculate the gas density by the ideal gas law, and the volume occupied by electrodes and other components is not considered. Gases are injected into the chamber through a shower head with diameter of $300 \mathrm{~mm}$. The chamber vacuum is maintained by a turbomolecular pump supported by a rotary-vane oil pump, and the base pressure is approximately on the order of $10^{-5}$ Torr.

A Hiden Analytical PSM QMS is mounted on the chamber sidewall and evacuated by a differential pumping unit consisting of a turbomolecular pump and a rotary-vane oil pump. The QMS chamber is maintained in vacuum on the order of $10^{-7}$ Torr. The orifice at the end of the QMS probe has a diameter of $150 \mu \mathrm{m}$ that separates the QMS chamber from the main chamber, keeping the pressure of the QMS 2 orders of magnitude lower than the main chamber. During our experiments, the ionizing electron energy, emission current, and secondary electron multiplier voltage of PSM are set to be $70 \mathrm{eV}, 1 \mu \mathrm{A}$, and 2100 $\mathrm{V}$, respectively.

The standard gas used in this work is a mixed gas of various noble gas species, consisting of $0.3854 \%$ of $\mathrm{Xe}, 4.6902 \%$ of $\mathrm{Kr}, 21.1039 \%$ of $\mathrm{He}$, and $73.8205 \%$ of $\mathrm{Ne}$, and its quality was certified by the Korea Research Institute of Standards and Science in Oct 2007 [19].

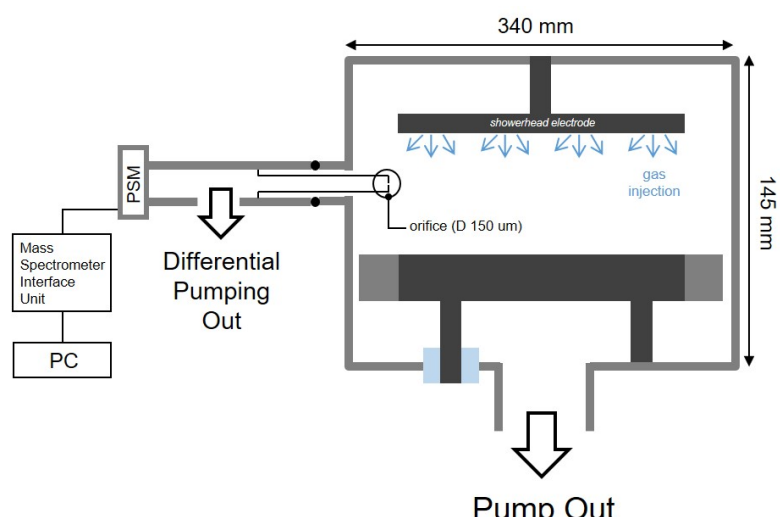

Figure 1. Schematic of the vacuum chamber equipped with QMS (Hiden, PSM).
The QMS data is acquired under various pressures 25 , 34, and 46 mTorr of the main plasma chamber. The experimental sequence is as follows: the standard gas is injected into the main chamber and a small amount of them is picked up to the QMS via orifice of it. Then, they are ionized and extracted to the quadrupole mass filter region, and the partial pressures of each mass are obtained through detector. According to a reported signal library for quadrupole mass spectrometry, the masses we consider to represent the partial pressures of each component of the standard gas are $4 \mathrm{amu}$ for $\mathrm{He}, 20 \mathrm{amu}$ for $\mathrm{Ne}, 84 \mathrm{amu}$ for $\mathrm{Kr}$, and 129, 131, and $132 \mathrm{amu}$ for Xe [20].

\section{Results and discussion}

Figure 2 shows the measured QMS signals of the standard gases under different pressures. According to Eq. (1), the differences between QMS signal intensities of each species can be caused by their MEs, ionization cross sections, and number density proportions. The filament emission current here is independent of gas chemistry. The ionization cross section of each species is $0.296,0.457,3.749$, and 4.67 in $10^{16} \mathrm{~cm}^{-3}$ for $\mathrm{He}, \mathrm{Ne}, \mathrm{Kr}$, and $\mathrm{Xe}$, respectively [21]. Even though the cross section of $\mathrm{Kr}$ and $\mathrm{Xe}$ are 10 times higher than that of $\mathrm{He}$ and $\mathrm{Ne}$, the relative number density of $\mathrm{Kr}$ and $\mathrm{Xe}$ is much lower $(10-100$ times) than that of $\mathrm{He}$ and $\mathrm{Ne}$, leading to the huge difference between the signal intensities of them.

By normalizing a signal intensity represented based on Eq. (1) of species $X(X=\mathrm{He}, \mathrm{Kr}$, or $\mathrm{Xe})$ with that of $\mathrm{Ne}$, we can obtain normalized ME (referred to $k_{X}$ ) for species $X$.

$$
\begin{aligned}
\frac{S_{X}}{S_{N e}} & =\frac{\alpha_{X}}{\alpha_{N e}} \frac{\sigma_{X}}{\sigma_{N e}} \frac{n_{X}}{n_{N e}} \\
& \equiv k_{X} \frac{\sigma_{X}}{\sigma_{N e}} \frac{n_{X}}{n_{N e}} .
\end{aligned}
$$

Figure 3 shows the calculated $k_{X}$ under various conditions. As shown in Fig. 2, the ME of each species is not significantly affected by pressure but a strong function of mass. Therefore, via the fitting process $k_{X}$ data with a mass function, we arrive at the simple result as follows,

$$
\operatorname{ME}(m)=2.4 \times m^{-0.44},
$$

where $m$ is the mass of each species. Eq. (4) can be applied for mass in the range of 4 to 131, where it is very common in processing using CxFx gases. The ME obtained by fitting our experimental data also showed the mass dependence that decreases with increasing mass, similar to that in refs. $[9,11,21]$, lower transmission probability for higher mass. This may be attributed to the longer travel time in the mass filter of a QMS of heavier particles that may indicate a higher

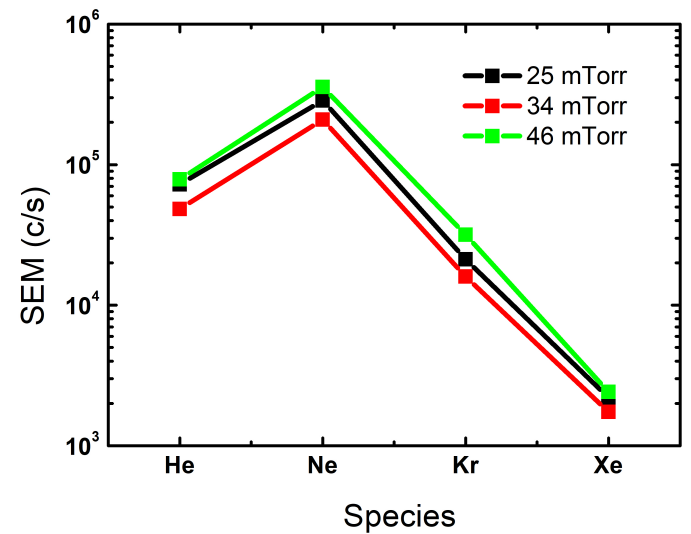

Figure 2. QMS signals of each component of the standard gas at different pressures. 


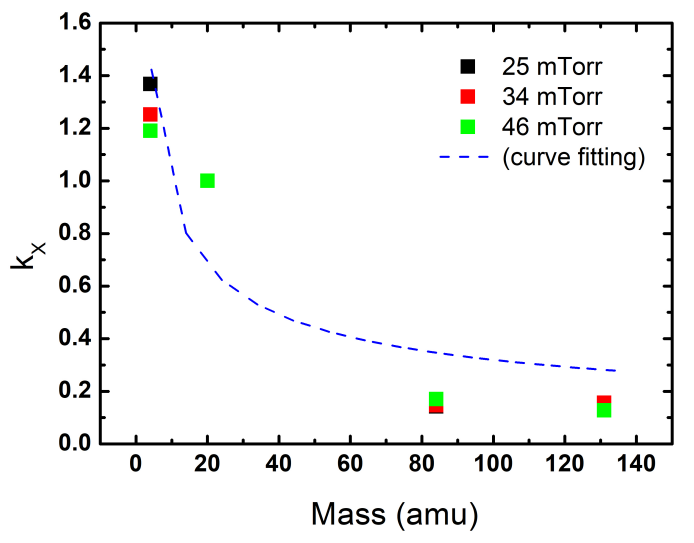

Figure 3. Normalized proportional coefficients of each component of the standard gas at different pressures and a fitting curve representing the normalized proportional coefficients as a function of a particle mass.

potential for particles to collide with background gas, which leads to a decrease in an ME.

We examined the proposed approach to quantify QMS signals by measuring Ar gas density in the vacuum chamber. Ar at $50 \mathrm{sccm}$ was injected into the chamber and the chamber pressure was controlled by a throttle valve. We obtained QMS data on the changes in a signal intenstiy of Ar with pressure increase. To verify the reliability of our method, the obtained QMS signals were converted to Ar density with various MEs: one obtained by the new approach and the others referred from previous research $[15,17]$. According to Eq. (4), $k_{X}$, which is the ME of Ar normalized with that of $\mathrm{Ne}$, is 0.47. Referring to [15], the ME is inversely proportional to a mass, leading to $k_{X}$ of 0.5 . Another $k_{X}$ value proposed by Singh et al. is the Ar-to-Ne mass ratio to the power of -0.81 , which is 0.57 . Using these different values of $k_{X}$, we estimated the Ar gas density in the chamber under different pressures; the comparison result is shown in Fig. 4. It should be noted that Ar densities under various pressures calculated by the ideal gas law are assumed to be true values.

With increasing pressure, the difference between the true values and estimated values also increases. This may be due to the fact that an increase in the QMS pressure raises collisions between the transmitting ions and background gases, leading to an increase in the error of the transmission efficiency. However, it is shown in Fig. 4 that our results are closer to the true values than the others.

Analysis on this comparison result is as follows: It is reasonable that the trajectory of an ion passing through the space of the quadrupole

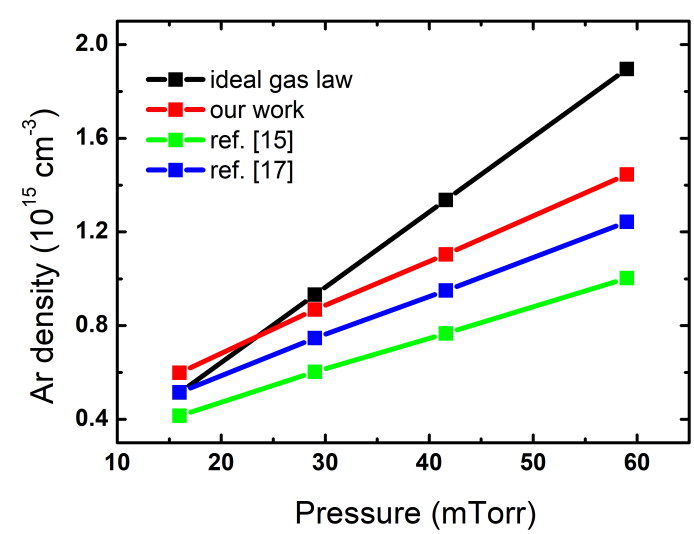

Figure 4. QMS data quantization result with the proposed approach (red) and with traditional approaches proposed in [15] (green) and [17] (blue), compared with calculated values by the ideal gas law (black).

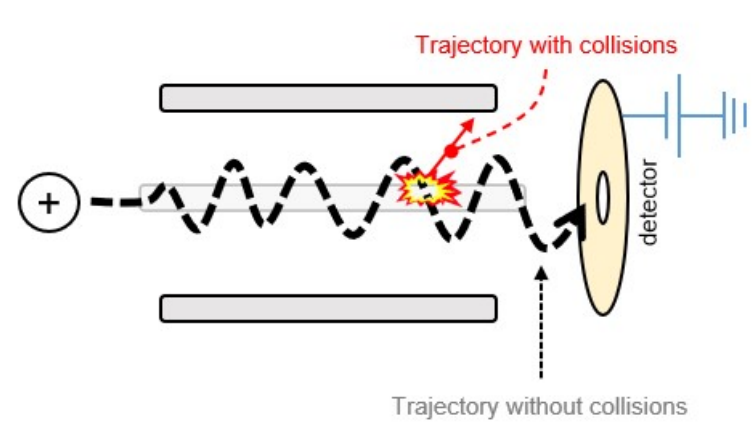

Figure 5. Illustration of an ion trajectory that changes according to the existence of collisions between the ion and background gas.

electrode can be distorted by collisions between the ion and the background gas, as shown in Fig. 5. The possibility of the collisions is approximately proportional to the transit time of the ion, which is inversely proportional to the ion velocity. In other words, the probability of the collisions $P$ is proportional to the ion velocity, as

$$
P \propto v^{-1} .
$$

Meanwhile, every ion that has entered the quadrupole region is accelerated by the same voltage applied to the detector (see Fig. 5). This means that all energies of ions traveling through the quadrupoles are even regardless of ion mass. Therefore, ion velocity is proportional to the inverse square root of the ion mass as shown below:

$$
E=\frac{1}{2} m v^{2}=\text { const. } \rightarrow v \propto m^{-1 / 2} .
$$

This leads to the relation between the probability of the collisions in the quadrupole region and the ion mass, which is $P \propto m^{1 / 2}$, which reflects the quadrupole transmittance is inversely proportional to ion mass $m$.

Koizumi and Chihara showed that the detection efficiency of a channelelectron multiplier, which corresponds to the type of the PSM detector used in our work, does not depend on both the mass and charge of an impinging ion in the energy regime of a few kilovolts [22], which is consistent with our PSM operation condition. Note that their result is surely different from that proposed by Kessels, et al., which is inversely proportional to the square root of mass.

We can now approximately obtain the relation between an ME and an ion mass,

$$
\mathrm{ME} \propto m^{-1 / 2} .
$$

It is noted that in the comparison result shown in Fig. 4 , the ME in our approach and previous research $[15,17]$ is proportional to the ion mass to the power of $-0.44,-1$, and -0.81 , respectively. Referring to the rough scaling in Eq. (7), we concluded that our work showed best estimation since its scaling factor, -0.44 , is closest to that in Eq. (7), -0.5. This indicates that MEs in QMS measurements even simply obtained using a standard gas may allow quantifying results to be improved.

\section{Conclusions}

We proposed a simple approach to obtain MEs of QMS signals with a standard gas. After measuring the QMS signals of all components of a standard gas and calculating the MEs using Eq. (1), one can obtain the MEs as a function of mass by fitting the calculated data. Quantification results with proposed MEs showed the best agreement with the results calculated by the ideal gas law, compared with those with different MEs from previous research. This reflects that MEs in QMS signals 
can simply be estimated using the QMS signals of standard gases. It should be noted that using standard gases consisting of some molecular gases may raise uncertainty in the measured QMS signals due to signal dispersion generated from molecular dissociation. Our results are expected to increase the reliability of data measured by QMSs, contributing an improvement to plasma database development.

\section{Acknowledgements}

This research was supported by the National Research Council of Science \& Technology (NST) grant by the Korea government (MSIP) (No. CAP-17-02-NFRI, CRF-20-01-NFRI), by the Industrial Strategic Technology Development Program-Next Generation Semiconductor R\&D (20010412, Development of Metal Oxide Carbon Layer Strip Process and Commercial Equipment for EUV Mask) funded by the Ministry of Trade, Industry \& Energy (MOTIE, Korea), by the Korea Institute of Energy Technology Evaluation and Planning (KETEP) and the MOTIE of the Republic of Korea (No. 20172010105910), by the MOTIE $(20009818,20010420)$ and KSRC(Korea Semiconductor Research Consortium) support program for the development of the future semiconductor device, by Korea Institute for Advancement of Technology (KIAT) grant funded by the Korea Government (MOTIE) (P0008458, The Competency Development Program for Industry Specialist), and by a Basic Science Research Program through the National Research Foundation of Korea (NRF) funded by the Ministry of Education (NRF-2020R1A6A1A03047771).

\section{References}

[1] I. Adamovich et al., J. Phys. D: Appl. Phys. 50, 323001 (2017)

[2] M. A. Lieberman and A. J. Lichtenberg, Principles of Plasma Discharges and Materials Processing, 2nd Ed. (John Wiley \& Sons, Inc., 2015).

[3] V. M. Donnelly and Avinoam Kornblit, J. Vac. Sci. Technol. A 31, 050825 (2013).
[4] K. J. Kanarik, T. Lill, E. A. Hudson, S. Sriraman, S. Tan, J. Marks, V. Vahedi, and R. A. Gottscho, J. Vac. Sci. Technol. A 33, 020802 (2015).

[5] G. S. Oehrlein, D. Metzler, and C. Li, ECS J. Solid State Sci. Technol. 4, N5041 (2015).

[6] T. Faraz, F. Roozeboom, H. C. M. Knoops, and W. M. M. Kessels, ECS J. Solid State Sci. Technol. 4, N5023 (2015).

[7] B. Wu, A. Kumar, and S. Pamarthy, J. Appl. Phys. 108, 051101 (2010).

[8] S. Huang, C. Huard, S. Shim, S. K. Nam, I. C. Song, S. Lu, and M. J. Kushner, J. Vac. Sci. Technol. A 37, 031304 (2019).

[9] J. W. Coburn and H. F. Winters, J. Appl. Phys. 50, 3189 (1979).

[10] H. C. Lee, Appl. Phys. Rev. 5, 011108 (2018).

[11] J. W. Coburn and M. Chen, J. Appl. Phys. 51, 3134 (1980).

[12] R. Engeln, B. Klarenaar, and O. Guaitella, Plasmas Sources Sci. Technol. 29, 063001 (2020).

[13] J. A. Simpson, Rev. Sci. Instrum. 32, 1283 (1961).

[14] H. J. Yeom, J. J. Lee, S. J. Kim, Y. S. Lee, C. H. Cho, J. H. Kim, H. C. Lee, and S. J. You, Appl. Sci. Converg. Technol. 29, 77 (2020).

[15] W. M. M. Kessels, C. M. Leewis, M. C. M. van de Sanden, and D. C. Schram, J. Appl. Phys. 86, 4029 (1999).

[16] H. Singh, J. W. Coburn, and D. B. Graves, J. Vac. Sci. Technol. A 17, 2447 (1999).

[17] H. Singh, J. W. Coburn, and D. B. Graves, J. Vac. Sci. Technol. A 18,299 (2000).

[18] W. S. Chang et al., Appl. Surf. Sci. 515, 145974 (2020).

[19] Korea Research Institute of Standards and Science www.kriss.re.kr (accessed Mar.8, 2021).

[20] N. Barros, J. Thurn, and K. Zuber, J. Phys. G: Nucl. Part. Phys. 41, 115105 (2014).

[21] J. H. Park, H. C. Choi, W. S. Chang, S. Y. Chung, D. C. Kwon, M. Y. Song, and J. S. Yoon, Appl. Sci. Converg. Technol. 29, 5 (2020).

[22] T. Koizumi and Y. Chihara, J. Phys.: Conf. Ser. 163, 012114 (2009). 